\title{
NEUMANN BOUNDARY CONDITIONS FOR A NONLOCAL NONLINEAR DIFFUSION OPERATOR. CONTINUOUS AND DISCRETE MODELS
}

\author{
MAURICIO BOGOYA, RAUL FERREIRA, AND JULIO D. ROSSI \\ (Communicated by David Tartakoff)
}

\begin{abstract}
Let $J: \mathbb{R} \rightarrow \mathbb{R}$ be a nonnegative, smooth function with $\int_{\mathbb{R}} J(r) d r=$ 1 , supported in $[-1,1]$, symmetric, $J(r)=J(-r)$, and strictly increasing in $[-1,0]$. We consider the Neumann boundary value problem for a nonlocal, nonlinear operator that is similar to the porous medium, and we study the equation

$$
u_{t}(x, t)=\int_{-L}^{L}\left(J\left(\frac{x-y}{u(y, t)}\right)-J\left(\frac{x-y}{u(x, t)}\right)\right) d y, \quad x \in[-L, L] .
$$

We prove existence and uniqueness of solutions and a comparison principle. We find the asymptotic behaviour of the solutions as $t \rightarrow \infty$ : they converge to the mean value of the initial data. Next, we consider a discrete version of the above problem. Under suitable hypotheses we prove that the discrete model has properties analogous to the continuous one. Moreover, solutions of the discrete problem converge to the continuous ones when the mesh parameter goes to zero. Finally, we perform some numerical experiments.
\end{abstract}

\section{INTRODUCTION}

Let $J: \mathbb{R} \rightarrow \mathbb{R}$ be a nonnegative, smooth function with $\int_{\mathbb{R}} J(r) d r=1$. Assume also that $J$ is supported in $[-1,1]$, is symmetric, $J(r)=J(-r)$, and strictly increasing in $[-1,0]$ (and hence strictly decreasing in $[0,1]$ ). Equations of the form

$$
u_{t}(x, t)=J * u-u(x, t)=\int_{\mathbb{R}} J(x-y) u(y, t) d y-u(x, t)
$$

and variations of it, have been recently widely used to model diffusion processes, see $[2],[3],[5],[6],[8],[9],[11]$. As stated in [8], if $u(x, t)$ is thought of as a density at the point $x$ at time $t$ and $J(x-y)$ is thought of as the probability distribution of jumping from location $y$ to location $x$, then $(J * u)(x, t)$ is the rate at which individuals are arriving to position $x$ from all other places and $-u(x, t)=-\int_{\mathbb{R}} J(y-x) u(x, t) d y$ is the rate at which they are leaving location $x$ to travel to all other sites. This consideration in the absence of external sources, leads immediately to the fact that

Received by the editors April 24, 2006.

2000 Mathematics Subject Classification. Primary 35K57, 35B40.

Key words and phrases. Nonlocal diffusion, Neumann boundary conditions.

The third author was supported by University de Buenos Aires under grant TX048, by ANPCyT PICT No. 00137 and by CONICET (Argentina), MB by MECESUP (Chile) and RF by BFM2002-04572 (Spain). 
the density $u$ satisfies equation (1.1). Equation (1.1), the so called nonlocal diffusion equation, shares many properties with the classical heat equation $u_{t}=\Delta u$, such as that a maximum principle holds for both of them and, even if $J$ is compactly supported, perturbations propagate with infinite speed.

Another classical equation that has been used to model diffusion is the wellknown porous medium equation, $u_{t}=\Delta u^{m}$ with $m>1$. This equation also shares several properties with the heat equation but there is a fundamental difference: in this case we are facing a nonlinear diffusion operator, diffusion depends on the density $u$. Properties of solutions for the porous medium equation have been studied intensively in the recent past. See for example [1], [10] and the corresponding bibliography.

A simple nonlocal nonlinear model for diffusion where the diffusion at a point depends on the density, as happens for the porous medium equation, was introduced in [7]. In this model the probability distribution of jumping from location $y$ to location $x$ is given by $J\left(\frac{x-y}{u(y, t)}\right) \frac{1}{u(y, t)}$ when $u(y, t)>0$ and 0 otherwise. In this case the rate at which individuals are arriving to position $x$ from all other places is $\int_{\mathbb{R}} J\left(\frac{x-y}{u(y, t)}\right) d y$ and the rate at which they are leaving location $x$ to travel to all other sites is $-u(x, t)=-\int_{\mathbb{R}} J\left(\frac{y-x}{u(x, t)}\right) d y$. As before and in the absence of external sources, this consideration leads immediately to the fact that the density $u$ has to satisfy

$$
u_{t}(x, t)=\int_{\mathbb{R}} J\left(\frac{x-y}{u(y, t)}\right) d y-u(x, t) .
$$

It is proved in [7] that this problem shares with the porous medium equation the finite speed of propagation property. Compactly supported initial data develop a free boundary and eventually cover the whole $\mathbb{R}$.

Our main concern in this paper is to look for Neumann boundary conditions for this nonlocal nonlinear diffusion operator.

We study the problem

$$
u_{t}(x, t)=\int_{-L}^{L}\left(J\left(\frac{x-y}{u(y, t)}\right)-J\left(\frac{x-y}{u(x, t)}\right)\right) d y
$$

in $[-L, L] \times[0, \infty)$ with an initial datum $u(x, 0)=u_{0}(x)$. In this model it is assumed that no individual can jump inside nor outside the domain $[-L, L]$; therefore, the integrals are considered in $[-L, L]$ instead of in the whole $\mathbb{R}$. This says that the flux of individuals leaving or entering the domain is null. This is what is usually known as Neumann boundary conditions. We use ideas from [7] to prove the following result, but the details are technically different.

Theorem 1.1. For every $u_{0} \in L^{1}([-L, L])$ with $u_{0} \geq 0$ there exists a unique solution $u$ of $(1.2)$ such that $u \in C\left([0, \infty) ; L^{1}([-L, L])\right)$. Continuous solutions have a comparison property, if $u_{0}(x) \leq v_{0}(x) \in C([-L, L])$, then $u(x, t) \leq v(x, t)$ in $[-L, L] \times[0, \infty)$, and preserve the total mass in $[-L, L]$; that is,

$$
\int_{-L}^{L} u(y, t) d y=\int_{-L}^{L} u_{0}(y) d y
$$


Moreover, if $u_{0} \in C^{1}([-L, L])$ is strictly positive, the following asymptotic behavior takes place:

$$
u(x, t) \rightarrow \frac{1}{2 L} \int_{-L}^{L} u_{0}(x) d x, \quad \text { as } t \rightarrow+\infty \quad \text { uniformly in }[-L, L] .
$$

This result is analogous to the well-known result for the porous medium equation, $u_{t}=\left(u^{m}\right)_{x x}$, with Neumann boundary conditions, $\left(u^{m}\right)_{x}( \pm L, t)=0$, where solutions are known to preserve the total mass and converge to the mean value of the initial datum.

Next, we study a discrete version of the previous equation. A discrete analog to (1.1) is considered, for example, in [4]. We consider a set of nodes $-L=x_{-N}<$ $\cdots<x_{i}=h i<\cdots<x_{N}=L, i=-N, \ldots, N$, and discretize the integrals involved. We obtain

$$
\left(u_{i}\right)^{\prime}(t)=\sum_{j=-N}^{N} h J\left(\frac{h(i-j)}{u_{j}(t)}\right)-\sum_{j=-N}^{N} h J\left(\frac{h(i-j)}{u_{i}(t)}\right)
$$

with $h>0$ and initial datum $u_{i}(0)=u_{0}\left(x_{i}\right)$. We use the discrete analog to the $L^{1}$, the space $l_{h}^{1}=\left\{\left(u_{i}\right)\right\}$ with the norm $\left\|u_{i}\right\|_{l_{h}^{1}}:=\sum_{i=-N}^{N} h\left|u_{i}\right|$, to obtain the following result.

Theorem 1.2. For every $u_{0} \geq 0$ there exists a unique solution in $C\left([0, \infty) ; l_{h}^{1}\right)$ of (1.3) which depends continuously on the initial datum. A comparison principle holds: if $u_{i}(0) \leq v_{i}(0)$, then $u_{i}(t) \leq v_{i}(t)$ for all $i=-N, \ldots, N, t>0$. The solution preserves the total mass, i.e.,

$$
\sum_{i=-N}^{N} u_{i}(t)=\sum_{i=-N}^{N} u_{i}(0)
$$

and satisfies

$$
u_{i}(t) \rightarrow \frac{1}{2 N+1} \sum_{i=-N}^{N} u_{i}(0), \quad \text { as } t \rightarrow+\infty .
$$

Moreover if $u(x, t)$ is a positive $C^{1}$ solution of $(1.2)$ and $u_{i}(t)$ is the solution of (1.3), then there exists a constant $C$ such that

$$
\max _{0<t<T} \sum_{i=-N}^{N} h\left|u\left(x_{i}, t\right)-u_{i}(t)\right| \leq C h .
$$

The paper is organized as follows. In Section 2 we deal with the continuous problem, in Section 3 with the discrete one, and finally in the last section we show some numerical experiments.

\section{The Continuous Problem}

The existence and uniqueness result will be a consequence of a fixed point theorem. Fix $t_{0}>0$ and consider the Banach space $C\left(\left[0, t_{0}\right] ; L^{1}([-L, L])\right)$ with the norm

Let

$$
|\|w\||=\max _{0 \leq t \leq t_{0}}\|w(\cdot, t)\|_{L^{1}([-L, L])} .
$$

$$
X_{t_{0}}=\left\{w \in C\left(\left[0, t_{0}\right] ; L^{1}([-L, L])\right) / w \geq 0\right\}
$$


which is a closed subset of $C\left(\left[0, t_{0}\right] ; L^{1}([-L, L])\right)$. We will obtain the solution as a fixed point of the operator $T_{w_{0}}: X_{t_{0}} \rightarrow X_{t_{0}}$ defined by

$$
T_{w_{0}}(w)(x, t)=\int_{0}^{t} \int_{-L}^{L}\left(J\left(\frac{x-y}{w(y, s)}\right)-J\left(\frac{x-y}{w(x, s)}\right)\right) d y d s+w_{0}(x) .
$$

The following lemma is the main ingredient of our proof.

Lemma 2.1. Let $w_{0}, z_{0}$ be nonnegative functions such that $w_{0}, z_{0} \in L^{1}([-L, L])$ and $w, z \in X_{t_{0}}$. Then there exists a constant $C$ such that

$$
\left\|\left|T_{w_{0}}(w)-T_{z_{0}}(z)\right||| \leq C t_{0}|||w-z|||+\right\| w_{0}-z_{0} \|_{L^{1}([-L, L])} .
$$

Proof. We have

$$
\begin{aligned}
& \int_{-L}^{L}\left|T_{w_{0}}(w)(x, t)-T_{z_{0}}(z)(x, t)\right| d x \\
& \leq \int_{0}^{t} \int_{-L}^{L}\left|\int_{-L}^{L}\left(J\left(\frac{x-y}{w(y, s)}\right)-J\left(\frac{x-y}{z(y, s)}\right)\right) d y\right| d x d s \\
& \quad+\int_{0}^{t} \int_{-L}^{L}\left|\int_{-L}^{L}\left(J\left(\frac{x-y}{w(x, s)}\right)-J\left(\frac{x-y}{z(x, s)}\right)\right) d y\right| d x d s \\
& +\int_{-L}^{L}\left|w_{0}-z_{0}\right|(y) d y
\end{aligned}
$$

To study the first term, we consider the sets $A^{+}(s)=\{y \in[-L, L] / w(y, s) \geq$ $z(y, s)\}$ and $A^{-}(s)=\{y \in[-L, L] / w(y, s)<z(y, s)\}$. We have

$$
\begin{aligned}
& \int_{-L}^{L}\left|\int_{-L}^{L}\left(J\left(\frac{x-y}{w(y, s)}\right)-J\left(\frac{x-y}{z(y, s)}\right)\right) d y\right| d x \\
& \leq \int_{-L}^{L} \int_{A^{+}(s)}\left(J\left(\frac{x-y}{w(y, s)}\right)-J\left(\frac{x-y}{z(y, s)}\right)\right) d y d x \\
& \quad+\int_{-L}^{L} \int_{A^{-}(s)}\left(J\left(\frac{x-y}{z(y, s)}\right)-J\left(\frac{x-y}{w(y, s)}\right)\right) d y d x .
\end{aligned}
$$

Since the integrands are nonnegative, we can apply Fubini's theorem to get

$$
\begin{aligned}
& \int_{-L}^{L} \int_{A^{+}(s)}\left(J\left(\frac{x-y}{w(y, s)}\right)-J\left(\frac{x-y}{z(y, s)}\right)\right) d y d x \\
& =\int_{A^{+}(s)}\left(w(y, s) \int_{\frac{-y-L}{w(y, s)}}^{\frac{-y+L}{w(y, s)}} J(r) d r-z(y, s) \int_{\frac{-y-L}{z(y, s)}}^{\frac{-y+L}{z(y, s)}} J(r) d r\right) d y \\
& \leq \int_{A^{+}(s)}(w(y, s)-z(y, s))\left(\int_{\frac{-y-L}{z(y, s)}}^{\frac{-y+L}{z(y, s)}} J(r) d r\right) d y \\
& \leq \int_{A^{+}(s)}|w(y, s)-z(y, s)| d y .
\end{aligned}
$$

We argue similarly with the integral over $A^{-}(s)$. Therefore, we obtain

$$
\int_{-L}^{L}\left|\int_{-L}^{L}\left(J\left(\frac{x-y}{w(y, s)}\right)-J\left(\frac{x-y}{z(y, s)}\right)\right) d y\right| d x \leq \int_{-L}^{L}|w(y, s)-z(y, s)| d y .
$$

The second term can be handled in the same way. Hence we get (2.1). 
Theorem 2.2. For every nonnegative $u_{0} \in L^{1}([-L, L])$ there exists a unique solution of $(1.2) u \in C\left([0, \infty) ; L^{1}([-L, L])\right)$. Moreover, the solution preserves the total mass, that is $\int_{-L}^{L} u(y, t) d y=\int_{-L}^{L} w_{0}(y) d y$.

Proof. From Lemma 2.1 we obtain that $T_{u_{0}}$ is a contraction in $X_{t_{0}}$ for $t_{0}$ small. Therefore, there exists a unique fixed point of $T_{u_{0}}$ in $X_{t_{0}}$. This provides us with a solution in $\left[0, t_{0}\right]$. To continue we may take as initial data $u\left(x, t_{0}\right)$ and obtain a solution in $\left[0,2 t_{0}\right]$. We continue this procedure to obtain a solution defined for all $t>0$. We finally prove that the integral in $x$ of $u$ is preserved. We have

$$
u(x, t)-u_{0}(x)=\int_{0}^{t} \int_{-L}^{L}\left(J\left(\frac{x-y}{u(y, t)}\right)-J\left(\frac{x-y}{u(x, t)}\right)\right) d y d s .
$$

We can integrate in $x$ and apply Fubini's theorem to obtain

$$
\begin{gathered}
\int_{-L}^{L} u(x, t) d x-\int_{-L}^{L} u_{0}(x) d x=\int_{0}^{t} \int_{-L}^{L} \int_{-L}^{L} J\left(\frac{x-y}{u(y, t)}\right) d x d y d s \\
-\int_{0}^{t} \int_{-L}^{L} \int_{-L}^{L} J\left(\frac{x-y}{u(x, t)}\right) d y d x d s=0 .
\end{gathered}
$$

This ends the proof of the theorem.

Remark 2.1. Solutions of (1.2) depend continuously on the initial condition in the following sense: if $u$ and $v$ are solutions of (1.2), then there exists a constant $C=C\left(t_{0}, J, L\right)$ such that

$$
\max _{0 \leq t \leq t_{0}}\|u(\cdot, t)-v(\cdot, t)\|_{L^{1}([-L, L])} \leq C\|u(\cdot, 0)-v(\cdot, 0)\|_{L^{1}([-L, L])} .
$$

Remark 2.2. If $u_{0} \geq \delta$ is $C^{k}$, then the solution $u(\cdot, t) \in C^{k}$ for all $t \geq 0$. Moreover, there exists a constant $C=C\left(\delta, J, u_{0}\right)$ such that $\left|u_{t}\right|,\left|u_{x}\right| \leq C$. This follows arguing as before but using the space $C\left(\left[0, t_{0}\right] ; C^{k}[-L, L]\right)$ instead of $C\left(\left[0, t_{0}\right] ; L^{1}[-L, L]\right)$.

Now we prove a comparison principle valid for continuous solutions.

Theorem 2.3. Let $u$ and $v$ be continuous solutions of $(1.2)$. If $u(x, 0) \leq v(x, 0)$ for all $x \in[-L, L]$, then $u(x, t) \leq v(x, t)$ for all $(x, t) \in[-L, L] \times[0, \infty)$.

Proof. We assume first that $u(x, 0)+\delta<v(x, 0)$. Moreover, we assume for a moment that $u(x, 0)$ and $v(x, 0)$ are $C^{1}$ functions. If the conclusion does not hold, we have that there exists a time $t_{0}>0$ and a point $x_{0} \in[-L, L]$ such that $u\left(x_{0}, t_{0}\right)=v\left(x_{0}, t_{0}\right)$ and $u(x, t) \leq v(x, t)$ for all $(x, t) \in[-L, L] \times\left[0, t_{0}\right]$.

Let us consider the set $B=\left\{x \in[-L, L] / u\left(x, t_{0}\right)=v\left(x, t_{0}\right)\right\}$. Clearly $B$ is nonempty and closed. Let $x_{1} \in B$. Then we have

$$
0 \leq(u-v)_{t}\left(x_{1}, t_{0}\right)=\int_{-L}^{L}\left(J\left(\frac{x_{1}-y}{u\left(y, t_{0}\right)}\right)-J\left(\frac{x_{1}-y}{v\left(y, t_{0}\right)}\right)\right) d y \leq 0,
$$

which implies $u\left(y, t_{0}\right)=v\left(y, t_{0}\right)$ for all $y \in B\left(x_{1}, r\right)$. Hence $B$ is open. It follows that $B=[-L, L]$, which is the desired contradiction.

We now get rid of the extra hypothesis that $w(x, 0)$ and $z(x, 0)$ are $C^{1}$ functions. In order to do this let $w_{n}(x, 0)$ and $z_{n}(x, 0)$ be sequences of $C^{1}$ functions such that $w_{n}(x, 0) \rightarrow w(x, 0)$ and $z_{n}(x, 0) \rightarrow z(x, 0)$ in $L^{1}([-L, L])$ as $n \rightarrow \infty$ and, moreover, $u_{n}(x, 0)=w_{n}(x, 0)<v_{n}(x, 0)=z_{n}(x, 0)$. Let $u_{n}$ and $v_{n}$ be the solutions with initial data $u_{n}(x, 0)$ and $v_{n}(x, 0)$, respectively. By the previous argument one has 
$u_{n} \leq v_{n}$ and the result follows by letting $n \rightarrow \infty$ in view of Remark 2.1 and the monotone convergence theorem.

We end this section studying the asymptotic behaviour. We prove that the solution converges to a constant and, since the total mass is preserved, this constant must be the mean value of the initial datum. Observe that only the steady states of the problem are constants. Also remark that this equation does not have any regularizing effect (see [7]) and, moreover, we cannot find a Lyapunov functional. Therefore, the asymptotic behaviour is not straightforward.

Theorem 2.4. Let $u_{0}$ be a positive $C^{1}$ function. Then

$$
u(x, t) \rightarrow \frac{1}{2 L} \int_{-L}^{L} u_{0}(x) d x, \quad \text { as } t \rightarrow+\infty \quad \text { uniformly in }[-L, L] .
$$

Proof. Let

$$
M(t)=\max _{x \in[-L, L]} u(x, t), \quad m(t)=\min _{x \in[-L, L]} u(x, t), \quad \text { and } \quad P=\frac{1}{2 L} \int_{-L}^{L} u_{0}(x) d x .
$$

From a comparison argument (constants are solutions of our problem) we get that $M$ is decreasing and bounded below by $P$ (by the conservation of mass). Therefore,

$$
\lim _{t \rightarrow \infty} M(t)=M_{\infty} \geq P .
$$

We claim that $M_{\infty}=P$. Assume that this is not the case. By our regularity assumptions on the initial datum, the solution is bounded in $C^{1}$, and from the conservation of the total mass for each $k$, there exists an interval $I_{k} \subset[-L, L]$ with $\left|I_{k}\right| \geq c$ where $u(x, k) \leq P$ for $x \in I_{k}$. Let $x_{k}$ be the midpoint of the interval $I_{k}$. By taking a subsequence if necessary, we may assume that $x_{k} \rightarrow x_{0}$. Let $I_{0}=\left(x_{0}-c / 2, x_{0}+c / 2\right) \cap[-L, L]$ and let $z(x, t)$ be the solution of the problem with initial datum $z(x, 0)$, a continuous function such that $M_{\infty}>z(x, 0) \geq P$ for $x \in I_{0}$ and $z(x, 0)=M_{\infty}$ for $x \in[-L, L] \backslash I_{0}$. We have that $\max _{x} z(x, 1)<M_{\infty}$. To prove this fact, just argue as in the proof of Theorem 2.3.

Now, we consider $z_{n}(x, t)$ the solution with initial datum $z_{n}(x, 0)=z(x, 0)+1 / n$. From continuous dependence of solutions with respect to the initial data we have that for $n_{0}$ large enough $\max _{x} z_{n_{0}}(x, 1)<M_{\infty}$.

On the other hand, for $k$ large enough we obtain that $u(x, k) \leq z_{n_{0}}(x, 0)$, then by a comparison argument we obtain

$$
M(k+1)=\max _{x} u(x, k+1) \leq \max _{x} z_{n}(x, 1)<M_{\infty},
$$

a contradiction that proves that $M(t) \rightarrow P$ as $t \rightarrow \infty$. Analogously, it can be proved that $m(t) \rightarrow P$ as $t \rightarrow \infty$. Hence,

$$
|u(x, t)-P| \leq \max \{M(t)-P, P-m(t)\} \rightarrow 0, \quad t \rightarrow \infty,
$$

as we wanted to prove.

\section{THE DISCRETE PROBLEM}

In this section we propose and analyze a discrete model for our nonlocal nonlinear diffusion operator. As we mentioned in the introduction, we consider a uniform 
mesh of the interval $[-L, L]$ composed by $x_{i}=i h, i=-N, \ldots, N$ and approximating the integrals in (1.2) we obtain

$$
\left(u_{i}\right)^{\prime}(t)=\sum_{j=-N}^{N} h J\left(\frac{h(i-j)}{u_{j}(t)}\right)-\sum_{j=-N}^{N} h J\left(\frac{h(i-j)}{u_{i}(t)}\right) .
$$

As initial datum we restrict $u_{0}$ to the mesh $u_{i}(0)=u_{0}\left(x_{i}\right)$.

As in the continuous case existence and uniqueness of the ODE's system (3.1) will be a consequence of Banach's fixed point theorem. Fix $t_{0}>0$ and consider the Banach space $C\left(\left[0, t_{0}\right] ; l_{h}^{1}\right)$ with the norm $|\|w\||=\max _{0 \leq t \leq t_{0}} \sum_{i=-N}^{N} h\left|w_{i}\right|(t)$. We also consider the closed subset $X_{t_{0}}^{h}=\left\{w \in C\left(\left[0, t_{0}\right] ; l_{h}^{1}\right) / w_{i} \geq 0\right\}$ and the operator $T_{w_{0}}^{h}: X_{t_{0}}^{h} \rightarrow X_{t_{0}}^{h}$ defined by

$$
\left(T_{w_{0}}^{h}(w)\right)_{i}(t)=\int_{0}^{t} \sum_{i=-N}^{N} h\left(J\left(\frac{h(i-j)}{w_{j}(s)}\right)-J\left(\frac{h(i-j)}{w_{i}(s)}\right)\right) d s+\left(w_{0}\right)_{i} .
$$

Now we prove a lemma similar to Lemma 2.1.

Lemma 3.1. For every $h>0$ there exists a constant $C=C(h)$ such that

$$
\left\|\left|T_{w_{0}}^{h}(w)-T_{z_{0}}^{h}(z)\right||| \leq C t_{0}\left|\left\|w-z\left|\|\mid+\| w_{0}-z_{0} \|_{l_{h}^{1}} .\right.\right.\right.\right.
$$

Proof. The proof is similar to that of Lemma 2.1. However a major difference appears: we do not have a change of variables formula like the one used in that proof. We overcome this difficulty by looking carefully at the size of the quantities involved. We have to deal with terms of the form

$$
\sum_{i=-N}^{N} h\left|\sum_{j=-N}^{N} h\left(J\left(\frac{h(i-j)}{w_{j}(s)}\right)-J\left(\frac{h(i-j)}{z_{j}(s)}\right)\right)\right| .
$$

Let $A^{+}(s)=\left\{j / w_{j}(s) \geq z_{j}(s)\right\}$ and $A^{-}(s)=\left\{j / w_{j}(s)<z_{j}(s)\right\}$. We can decompose the sum according to $j \in A^{+}(s)$ or $j \in A^{-}(s)$. Let us analyze the case $j \in A^{+}(s)$. Let $(i-j)=k$ and assume that $w_{j}(s)>z_{j}(s)>h|k|$, we obtain an upper bound for this part of the sum as follows,

$$
\begin{aligned}
& \sum_{j \in A^{+}(s)} h \sum_{k=-N-j}^{N-j} h\left(J\left(\frac{h k}{w_{j}(s)}\right)-J\left(\frac{h k)}{z_{j}(s)}\right)\right) \\
= & \sum_{j \in A^{+}(s)} h \sum_{k=-N-j}^{N-j} h J^{\prime}(\xi) h|k|\left(\frac{z_{j}(s)-w_{j}(s)}{w_{j}(s) z_{j}(s)}\right) \\
& \leq C\left(\sum_{k=-2 N}^{2 N} \frac{1}{|k|}\right)\left(\sum_{j \in A^{+}(s)} h\left(w_{j}(s)-z_{j}(s)\right)\right) .
\end{aligned}
$$

If $w_{j}(s)>h|k| \geq z_{j}(s)$ we have $J\left(\frac{h k}{z_{j}(s)}\right)=0$ and as $J(1)=0$, we obtain the bound

$$
\begin{gathered}
\sum_{j \in A^{+}(s)} h \sum_{k=-N-j}^{N-j} h\left(J\left(\frac{h k}{w_{j}(s)}\right)-J(1)\right)=\sum_{j \in A^{+}(s)} h \sum_{k=-N-j}^{N-j} h J^{\prime}(\xi) \frac{h|k|-w_{j}(s)}{w_{j}(s)} \\
\leq C\left(\sum_{k=-2 N}^{2 N} \frac{1}{|k|}\right)\left(\sum_{j \in A^{+}(s)} h\left(w_{j}(s)-z_{j}(s)\right)\right) .
\end{gathered}
$$


In the case $h|k| \geq z_{j}(s), w_{j}(s)$ the terms that appear in the sum vanish and there is nothing to deal with. From this point the rest of the proof runs as before.

With the inequality (3.2), it is easy to prove the existence and uniqueness of a solution. Moreover, the same arguments used in the continuous case provide a comparison principle and the asymptotic behaviour which is similar to the continuous one. That is, solutions converge to the mean value of the initial data.

Theorem 3.2. For every positive $u_{0} \in l_{h}^{1}$ there exists a unique solution of (3.1) in $C\left([0, \infty) ; l_{h}^{1}\right)$ which depends continuously on the initial datum. The solution preserves the total mass, that is

$$
\sum_{i=-N}^{N} u_{i}(t)=\sum_{i=-N}^{N} u_{i}(0)
$$

Moreover, solutions have a comparison principle: if $u_{i}(0) \leq v_{i}(0)$, then $u_{i}(t) \leq v_{i}(t)$ for all $i=-N, \ldots, N, t>0$, and satisfy

$$
u_{i}(t) \rightarrow \frac{1}{2 N+1} \sum_{i=-N}^{N} u_{i}(0), \quad t \rightarrow+\infty .
$$

To end this section, we study the convergence of the discrete solutions to the continuous ones as the mesh parameter $h$ goes to zero. We will restrict ourselves to strictly positive $C^{1}$ solutions.

Theorem 3.3. Let $u(x, t)$ be a positive $C^{1}$ solution of (1.2) and $u_{i}(t)$ the solution of (3.1). Then there exists a constant $C=C(J, T, L)$ such that

$$
\left\|\left|u\left(x_{i}, t\right)-u_{i}(t) \|\right| \leq C h \quad \forall t \in[0, T] .\right.
$$

Proof. Since the initial datum is positive, a comparison argument shows that there exists $\delta>0$ such that

$$
u(x, t), u_{h}(x, t) \geq \delta>0 .
$$

First, let us prove that the approximate scheme is consistent. To do that we only observe that for a $C^{1}$ function $f$,

$$
\int_{x_{j}}^{x_{j+1}} f(y) d y=h f\left(x_{j}\right)+O\left(h^{2}\right) .
$$

Notice that, as $J, u \in C^{1}$ and (3.4), the function $f(y)=J\left(\frac{x-y}{u(y, t)}\right)$ is a $C^{1}$ function. Then $v_{i}(t)=u\left(x_{i}, t\right)$ verifies that

$$
v_{i}^{\prime}(t)=\sum_{j=-N}^{N} h J\left(\frac{h(i-j)}{v_{j}(t)}\right)-\sum_{j=-N}^{N} h J\left(\frac{h(i-j)}{v_{i}(t)}\right)+O(h) .
$$

Therefore,

$$
\begin{gathered}
\int_{0}^{t}\left(u_{i}-v_{i}\right)^{\prime} d s=\int_{0}^{t} \sum_{j=-N}^{N} h\left(J\left(\frac{h(i-j)}{u_{j}(s)}\right)-J\left(\frac{h(i-j)}{v_{j}(s)}\right)\right) d s \\
-\int_{0}^{t} \sum_{j=-N}^{N} h\left(J\left(\frac{h(i-j)}{u_{i}(s)}\right)-J\left(\frac{h(i-j)}{v_{i}(s)}\right)\right) d s+O(h) .
\end{gathered}
$$


From the mean value theorem, the regularity of $J$, and (3.4) we obtain

$$
\left|\sum_{j=-N}^{N} h\left(J\left(\frac{h(i-j)}{u_{j}(s)}\right)-J\left(\frac{h(i-j)}{v_{j}(s)}\right)\right)\right| \leq C \sum_{j=-N}^{N} h\left|u_{j}-v_{j}\right|,
$$

where the constant $C=C(J, \delta)$ does not depend on $h$. A similar argument applied to the second term gives us

$$
\|u-v\|\left|-\|u(0)-v(0)\|_{l_{h}^{1}} \leq C_{1} t_{0}\left\||u-v \||+C_{2} t_{0} h .\right.\right.
$$

Since $C_{i}$ does not depend on $h$, we select $t_{0}$ also independent on $h$ such that $C_{1} t_{0}=1 / 2$. A continuation argument gives (3.3) for all $0<t<T$.

\section{Numerical EXPERIMENTS}

In this section we show some numerical experiments. We integrate (1.3) with an adaptive ODE solver using Matlab. We choose $L=2, u_{0}(x)=\max \{0,-x\}$ and $N=100$. We observe the evolution of the free boundary until the support reaches $x=L$ and the convergence to the mean value of the initial datum as $t \rightarrow \infty$. In Figure 1, the first picture shows the evolution of $u_{i}(t)$ and the second one the profiles of the solution at different times.

Finally, to see the convergence rate, we compute the difference between two discrete solutions for different values of $N$. We choose a strictly positive initial datum $u_{0}(x)=5-x^{2}, T=5$ and the norm

$$
\left\|u^{N}-u^{400}\right\|_{l_{h}^{1}}(T)=\sum_{i} h\left|\left(u^{N}\right)_{i}(T)-\left(u^{400}\right)_{i}(T)\right| .
$$
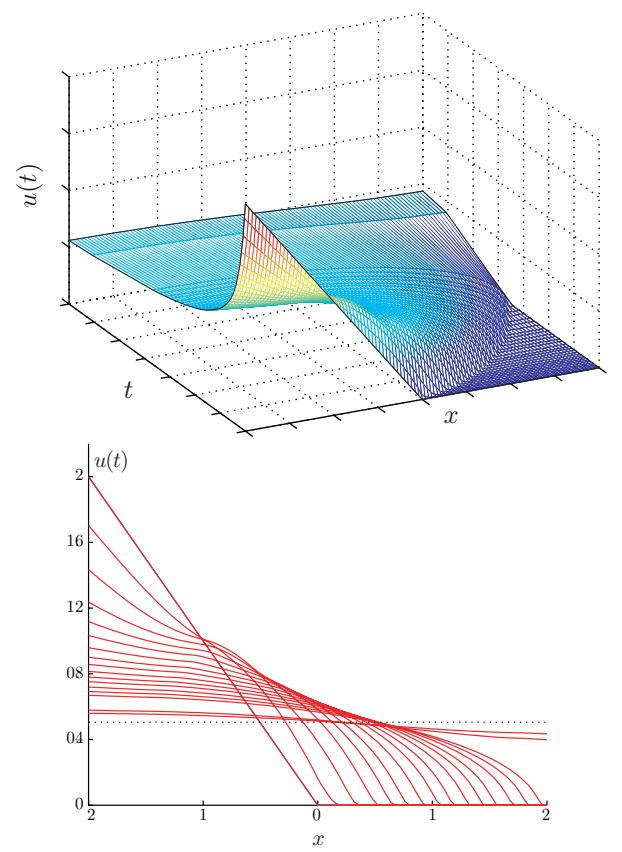

Figure 1 
We obtain the following table:

\begin{tabular}{c|cccc}
$N$ & 25 & 50 & 100 & 200 \\
\hline$\left\|u^{N}-u^{400}\right\|$ & 0.1358 & 0.0162 & 0.0044 & $6.3786 e-004$
\end{tabular}

As can be observed, the error decreases with $N$.

\section{REFERENCES}

[1] D. G. Aronson. The porous medium equation, in Nonlinear Diffusion Problems (A. Fasano and M. Primicerio eds.), Lecture Notes in Math., 1224, Springer Verlag, (1986). MR877986 (88a:35130)

[2] P. Bates and F. Chen. Spectral analysis and multidimensional stability of travelling waves for nonlocal Allen-Cahn equation. J. Math. Anal. Appl., 273, 45-57, (2002). MR1933014 (2003h:35104)

[3] P. Bates and A. Chmaj. An integrodifferential model for phase transitions: Stationary solutions in higher dimensions. J. Statistical Phys., 95, 1119-1139, (1999). MR1712445 (2000j:82020)

[4] P. Bates and A. Chmaj. A discrete convolution model for phase transitions. Arch. Rat. Mech. Anal., 150, 281-305, (1999). MR1741258 (2001c:82026)

[5] P. Bates, P. Fife, X. Ren and X. Wang. Travelling waves in a convolution model for phase transitions. Arch. Rat. Mech. Anal., 138, 105-136, (1997). MR1463804 (98f:45004)

[6] X. Chen. Existence, uniqueness and asymptotic stability of travelling waves in nonlocal evolution equations. Adv. Differential Equations, 2, 125-160, (1997). MR1424765 (98f:35069)

[7] C. Cortazar, M. Elgueta and J. D. Rossi. A non-local diffusion equation whose solutions develop a free boundary. Annales Henri Poincaré, 6(2), 269-281, (2005). MR2136191 (2006i:35379)

[8] P. Fife. Some nonclassical trends in parabolic and parabolic-like evolutions. Trends in nonlinear analysis, 153-191, Springer, Berlin, 2003. MR1999098 (2004h:35100)

[9] C. Lederman and N. Wolanski. Singular perturbation in a nonlocal diffusion model. Comm. Part. Differential Equations, 31(2), 195-241, (2006). MR2209752 (2007e:35166)

[10] J. L. Vazquez. An introduction to the mathematical theory of the porous medium equation, in Shape Optimization and Free Boundaries (M. C. Delfour ed.), Dordrecht, Boston and Leiden. 347-389, 1992. MR1260981 (95b:35101)

[11] X. Wang. Metastability and stability of patterns in a convolution model for phase transitions. J. Differential Equations, 183, 434-461, (2002). MR1919786 (2003f:35157)

Depto. de Matemática, Univ. Católica de Chile, Santiago, Chile

Current address: Depto. de Matemática, Univ. Nacional de Colombia, Bogotá, Colombia

E-mail address: mbogoyal@unal.edu.co

Depto. de Matemática, U. Carlos III, 28911, Leganés, España

E-mail address: raul.ferreira@uc3m.es

IMAFF, CSIC, SERrano 117, MADrID, España

Current address: Depto. Matematica, FCEyN, UBA, Buenos Aires, Argentina

E-mail address: jrossi@dm.uba.ar 